\title{
Usefulness of Simultaneous Magnetic Resonance Neurography and Apparent T2 Mapping for the Diagnosis of Cervical Radiculopathy
}

\author{
Keigo Enomoto ${ }^{1, *}$, Yawara Eguchi ${ }^{1,2,{ }^{*}}$, Takashi Sato ${ }^{1}$, Masaki Norimoto ${ }^{1}$, Masahiro Inoue ${ }^{3}$, \\ Atsuya Watanabe ${ }^{3}$, Takayuki Sakai ${ }^{4,5}$, Masami Yoneyama ${ }^{6}$, Yasuchika Aoki ${ }^{3}$, Sumihisa Orita ${ }^{1}$, \\ Miyako Narita ${ }^{1}$, Kazuhide Inage ${ }^{1}$, Yasuhiro Shiga ${ }^{1}$, Tomotaka Umimura ${ }^{1}$, Masashi Sato ${ }^{1}$, \\ Masahiro Suzuki ${ }^{1}$, Hiromitsu Takaoka ${ }^{1}$, Norichika Mizuki ${ }^{1}$, Geundong Kim ${ }^{1}$, Takashi Hozumi ${ }^{1}$, \\ Naoya Hirosawa ${ }^{1}$, Takeo Furuya ${ }^{1}$, Satoshi Maki ${ }^{1}$, Junichi Nakamura ${ }^{1}$, Shigeo Hagiwara ${ }^{1}$, Masao Koda ${ }^{7}$, \\ Tsutomu Akazawa ${ }^{8}$, Hiroshi Takahashi ${ }^{7}$, Kazuhisa Takahashi ${ }^{1}$, Seiji Ohtori ${ }^{1}$ \\ ${ }^{1}$ Department of Orthopaedic Surgery, Graduate School of Medicine, Chiba University, Chiba, Japan \\ ${ }^{2}$ Department of Orthopaedic Surgery, Shimoshizu National Hospital, Yotsukaido, Japan \\ ${ }^{3}$ Department of Orthopaedic Surgery, Eastern Chiba Medical Center, Togane, Japan \\ ${ }^{4}$ Department of Radiology, Eastern Chiba Medical Center, Togane, Japan \\ ${ }^{5}$ Faculty of Health Sciences, Institute of Medical, Pharmaceutical and Health Sciences, Kanazawa University, Kanazawa, Japan \\ ${ }^{6}$ MR Clinical Science, Philips Japan, Tokyo, Japan \\ ${ }^{7}$ Department of Orthopedic Surgery, University of Tsukuba, Tsukuba, Japan \\ ${ }^{8}$ Department of Orthopaedic Surgery, St. Marianna University School of Medicine, Kawasaki, Japan
}

Study Design: Retrospective observational study.

Purpose: We investigated the correlation between T2 relaxation times and clinical symptoms in patients with cervical radiculopathy caused by cervical disk herniation.

Overview of Literature: There are currently no imaging modalities that can assess the affected cervical nerve roots quantitatively. Methods: A total of 14 patients with unilateral radicular symptoms and five healthy subjects were subjected to simultaneous apparent T2 mapping and neurography with nerve-sheath signal increased with inked rest-tissue rapid acquisition of relaxation enhancement signaling (SHINKEI-Quant) using a 3-Tesla magnetic resonance imaging. The Visual Analog Scale (VAS) score for neck pain and upper arm pain was used to evaluate clinical symptoms. T2 relaxation times of the cervical dorsal root ganglia of the brachial plexus were measured bilaterally from C4 to C8 in patients with radicular symptoms and from C5 to C8 in healthy controls. The T2 ratio was calculated as the affected side to unaffected side.

Results: When comparing nerve roots bilaterally at each spinal level, no significant differences in T2 relaxation times were found between patients and healthy subjects. However, $\mathrm{T2}$ relaxation times of nerve roots in the patients with unilateral radicular symptoms were significantly prolonged on the involved side compared with the uninvolved side $(p<0.05)$. The VAS score for upper arm pain was not significantly correlated with the $\mathrm{T} 2$ relaxation times, but was positively correlated with the T2 ratio.

Received Dec 28, 2020; Revised Mar 15, 2021; Accepted Mar 18, 2021

Corresponding author: Yawara Eguchi

Department of Orthopaedic Surgery, Center for Orthopaedic Science Medical Innovation, Graduated School of Medicine, Chiba University, 1-8-1 Inohana, Chuo-ku, Chiba, 260-8670, Japan

Tel: +81-43-226-2117, Fax: +81-43-226-2116, E-mail: cafa0892@chiba-u.jp

*These two authors contributed equally to this work. 
Conclusions: In patients with cervical radiculopathy, the SHINKEI-Quant technique can be used to quantitatively evaluate the compressed cervical nerve roots. The VAS score for upper arm pain was positively correlated with the T2 ratio. This suggests that the SHINKEI-Quant is a potential tool for the diagnosis of cervical nerve entrapment.

Keywords: Cervical radiculopathy; Cervical disc herniation; Magnetic resonance imaging; Neurography

\section{Introduction}

Cervical radiculopathy is a familiar spinal condition caused by cervical nerve root compression [1]. Clinical symptoms of radiculopathy include neck and shoulder pain, radial pain along the distribution of the nerve roots, paresthesia, and weakness. The most frequent cause of cervical radiculopathy is nerve root entrapment in the intervertebral foramen caused by cervical spondylosis and disk herniation [2]. Although $40 \%$ to $80 \%$ of patients with cervical radiculopathy exhibit a good response after conservative treatment, some patients experience persistent radicular pain and progressive muscle weakness after conservative treatment and require surgery [3].

To treat cervical radiculopathy, it is necessary to accurately identify nerve compression lesions using clinical neurologic and diagnostic imaging techniques. Various imaging modalities can be used to assist with the diagnosis, including plain X-rays [4], computed tomography (CT) [5], CT myelography [6], and magnetic resonance imaging (MRI) [7].

There are currently no imaging modalities that can assess the affected cervical nerve roots quantitatively. Magnetic resonance (MR) neurography is a procedure that contributes to the visualization of the cervical nerve roots. Takahara et al. [8] and Yamashita et al. [9] successfully used MR neurography with diffusion-weighted imaging to examine the brachial plexus.

The three-dimensional (3D) nerve-sheath signal increased with inked rest-tissue rapid acquisition of relaxation enhancement imaging (SHINKEI) [10] is a new kind of MR neurography that suppresses signals from blood vessels, muscles, and fat using improved motionsensitized driven equilibrium (iMSDE) and spectral attenuated inversion recovery [10-14]. SHINKEI can morphologically evaluate the cervical nerve roots. However, it does not allow a quantitative assessment of the nerve roots involved. Recently, Yoneyama et al. [15] reported that SHINKEI-Quant can acquire MR neurography and simultaneously evaluate $\mathrm{T} 2$ relaxation times quantitatively. Hiwatashi et al. [16] reported that patients with chronic inflammatory demyelinating polyneuropathy (CIDP) can be distinguished from healthy subjects using SHINKEIQuant for the brachial plexus [16] and lumbar plexus [17].

Eguchi et al. [18] previously reported that, in a patient with cervical radiculopathy caused by intervertebral disk herniation before microendoscopic surgery, neurography disclosed swelling of the compressed nerve and a longer $\mathrm{T} 2$ relaxation time than that of the unaffected contralateral nerve. Furthermore, the approach using the SHINKEIQuant technique was successful in identifying lumbar radiculopathy [19]. The T2 ratio, defined as the T2 relaxation time on the affected side to unaffected side, was associated with leg pain in patients with lumbar radiculopathy. However, there are no reports that describe whether T2 relaxation time correlates with clinical symptoms in cervical radiculopathy. We hypothesized that SHINKEIQuant can identify the compressed cervical nerve roots of patients with radiculopathy using a quantitative approach. This study aimed to determine the relationship between $\mathrm{T} 2$ relaxation times and clinical symptoms using SHINKEI-Quant in patients with cervical radiculopathy caused by cervical disk herniation.

\section{Materials and Methods}

\section{Participants}

An informed consent was obtained from all participants before study initiation. All procedures performed in the studies involving human participants were in accordance with the ethical standards of the institutional and/or national research committee and with the 1964 Helsinki Declaration and its later amendments or comparable ethi- 
cal standards. The institutional ethics review committee of Shimoshizu National Hospital approved the study protocol (IRB approval no., H25'-1).

A total of 14 patients (eight males and six females; mean age, 53.0 \pm 9.84 years; range, $40-72$ years) with unilateral radicular symptoms owing to cervical disk herniation that involved arm pain and numbness and five healthy subjects (all males; mean age, 37.0 \pm 7.0 years; range, 32-45 years) were subjected to concurrent apparent T2 mapping and neurography with SHINKEI-Quant. The patients were diagnosed based on both neurologic symptoms and diagnostic images from plain radiographs, CT, and MRI. The average interval between the onset of symptoms and the first visit was $7.1 \pm 8.5$ weeks. Notably, one patient was affected at the $\mathrm{C} 4$ level, one patient at $\mathrm{C} 5$, eight patients at $\mathrm{C} 6$, three patients at $\mathrm{C} 7$, and one patient at $\mathrm{C} 8$; four microendoscopic surgeries and one laminoplasty were performed. The exclusion criteria were patients who had (1) bilateral symptoms and polyradiculopathy, (2) cervical canal stenosis, (3) cervical spine surgery before the study began, and (4) a spinal tumor, infectious disease, or spinal trauma.

The clinical symptoms were assessed using the Visual Analog Scale (VAS) score for neck pain and upper arm pain, ranging from 100 (extreme pain) to 0 (no pain). The $\mathrm{T} 2$ ratio was calculated as the $\mathrm{T} 2$ relaxation time on the affected side to unaffected side. The clinical evaluations were conducted before surgery and MRI.

\section{SHINKEI-Quant imaging technique}

\section{1) Image acquisition}

The SHINKEI-Quant imaging technique was used for a thorough examination of the affected nerves. A 3.0 T system (Ingenia CX; Philips Medical Systems, Amsterdam, The Netherlands) with a dStream TotalSpine coil was used to perform MRI for all subjects. Simultaneous T2 mapping and neurography with SHINKEI-Quant were acquired in the coronal plane according to the brachial plexus MR neurography protocol [20]. SHINKEIQuant details have been described elsewhere [10-14]. In brief, it is a turbo spin echo imaging approach that uses a diffusion-weighted pre-pulse called iMSDE to suppress signals from vessels and muscles and a short tau inversion recovery pre-pulse that suppresses the fat signal. This is followed by a readout procedure with a tissue-specific variable refocusing flip-angle rapid acquisition and a relaxation enhancement sequence to obtain contrastefficient T2 weighting [10]. To simultaneously synthesize MR neurography and estimate the apparent $\mathrm{T} 2$ relaxation time, two different iMSDE preparation times (sequences 1 and 2) were interwoven by repetition time (TR) into a single acquisition. SHINKEI-Quant parameters included TR/echo time (TE) of 2,400/47 ms, field of view of $300 \times 384 \mathrm{~mm}$, echo train length of 90 , acquisition matrix of $224 \times 287$, acquisition voxel size of $1.34 \times 1.34 \times 4.00 \mathrm{~mm}$, reconstructed voxel size of $0.75 \times 0.75 \times 2.00 \mathrm{~mm}^{3}$, b of 10 $\mathrm{sec} / \mathrm{mm}^{2}$, iMSDE prep-time of $36 \mathrm{~ms}$ for sequence 1 and $72 \mathrm{~ms}$ for sequence 2 , and acquisition time of 7 minutes and 12 seconds.

\section{2) Image segmentation}

MRI data were transferred to a personal computer, and Extended MR Workspace software (Philips Medical Systems) was used for T2 mapping. Circular regions of interest (ROIs, $25-50 \mathrm{~mm}^{2}$ ) were defined bilaterally at the cervical dorsal root ganglia (DRG) of the brachial plexus at $\mathrm{C} 4-\mathrm{C} 8$ in patients and at $\mathrm{C} 5-\mathrm{C} 8$ in healthy individuals by two spine surgeons with a minimum of 10 years of experience (Y.E. and K.E.). Investigators were blinded to the affected side and the presence of clinical symptoms. MR neurography and the T2 map were evaluated anatomically from the same slice. The bilateral circular ROIs that excluded the outside tissue on neurography were copied to a T2 map to measure T2 relaxation times (Zio station2; AMIN, Tokyo, Japan). Intraobserver reliability (Y.E.) and interobserver reliability (Y.E. versus K.E.) were calculated. We considered a statistical correlation when the $r$-value was $\geq 0.3$ or $<-0.3$. A $p$-value of $<0.05$ was considered statistically significant.

\section{Statistical analyses}

All data are expressed as mean \pm standard deviation. A paired $t$-test was used to compare T2 values between the right and left sides for each DRG from $\mathrm{C} 4$ to $\mathrm{C} 8$ in radiculopathy patients and $\mathrm{C} 5$ to $\mathrm{C} 8$ in healthy subjects. Correlations were calculated between the T2 relaxation times or T2 ratios and clinical symptoms such as the VAS scores using Pearson product-moment correlation. Spearman's correlation coefficients were used to test intra- and interindividual reliabilities. SAS ver. 9.4 for Windows (SAS Institute Inc., Cary, NC, USA) was used for analyses. A $p<0.05$ was considered statistically significant. 


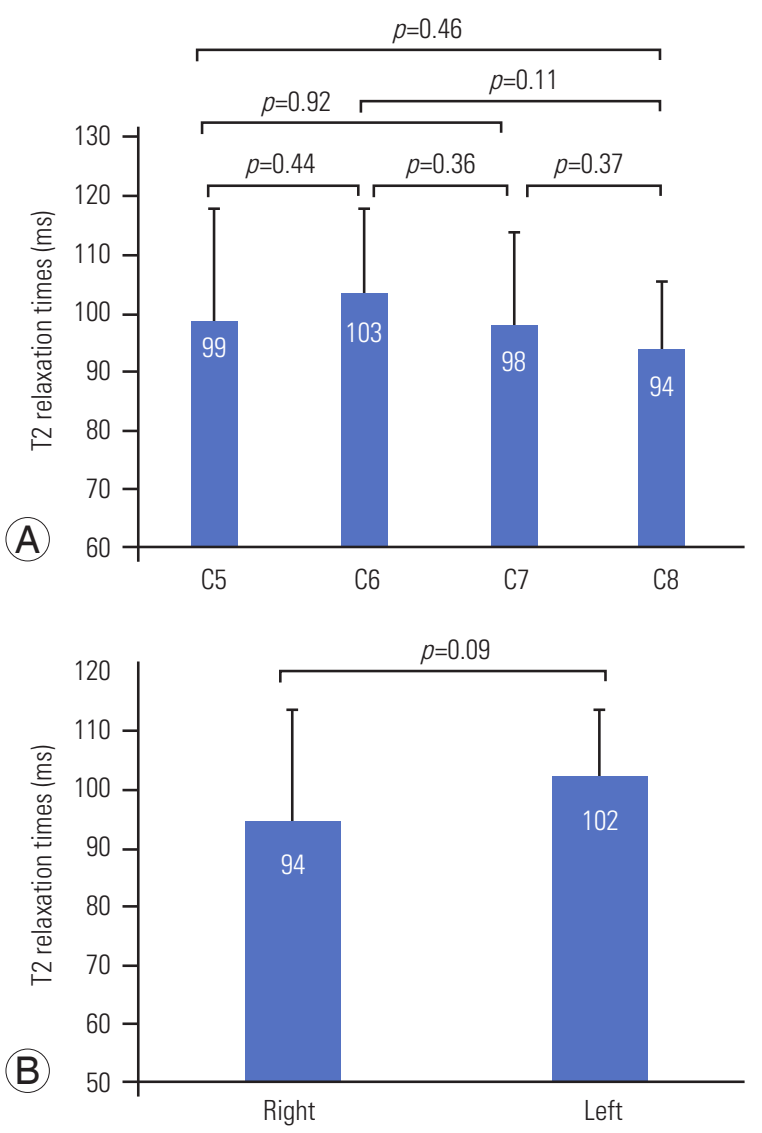

Fig. 1. Cervical nerve dorsal root ganglia T2 relaxation times in healthy individuals. (A) T2 relaxation times (ms) at each spinal level in healthy subjects. No significant differences in nerve root $\mathrm{T} 2$ relaxation times were identified among the spinal levels between C5 and C8 in healthy subjects. (B) Bilateral T2 relaxation times (ms) for nerve roots at each spinal level in healthy subjects (right side, left side). No significant differences were found between the nerve roots on the left and right sides $(p=0.09)$.

\section{Results}

\section{Main results}

There were no significant differences in the $\mathrm{T} 2$ relaxation times among spinal levels from C5 to C8 in healthy individuals. Likewise, there were no significant differences in T2 relaxation times (ms) between left and right DRGs at each spinal level ( $p=0.09$ ) (Fig. 1).

Intraobserver and interobserver reliabilities for T2 values were good ( $r=0.77$ and $r=0.78$, respectively).

In contrast, T2 relaxation times (ms) of DRGs in the patients with unilateral radicular symptoms were significantly prolonged on the involved side $(p<0.05)$ (Fig. 2).

The VAS score for neck pain was not significantly cor-

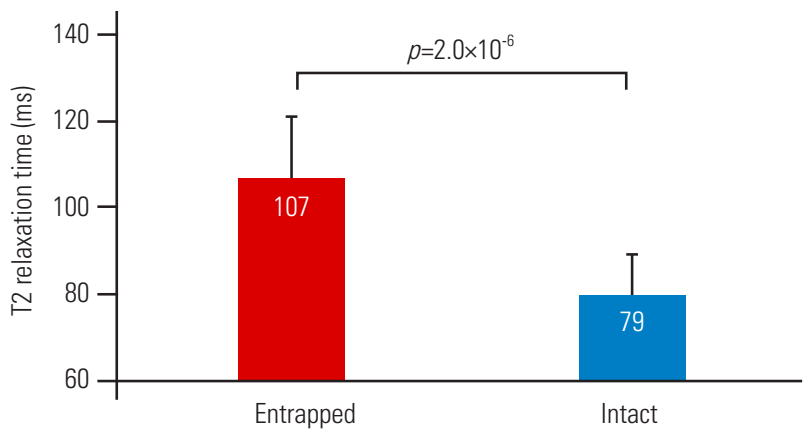

Fig. 2. Cervical nerve dorsal root ganglia (DRG) T2 relaxation times in patients with cervical radiculopathy. T2 relaxation times of DRGs in the patients with unilateral radicular symptoms were significantly prolonged on the involved side $(p<0.05)$.
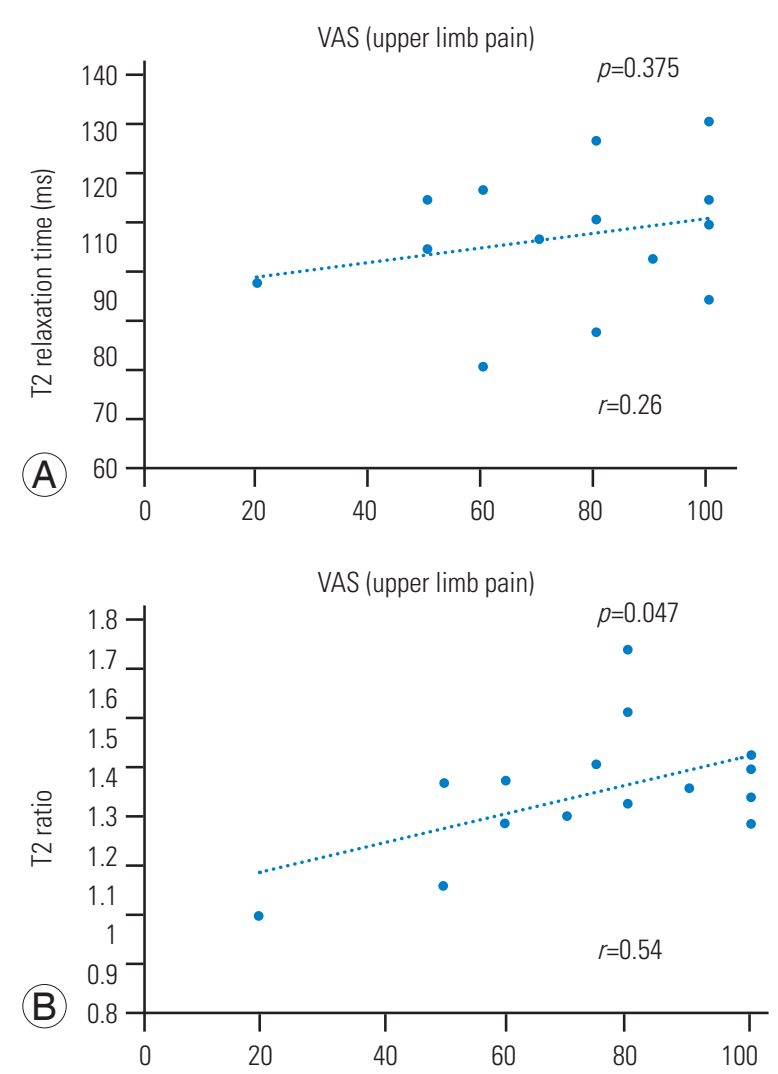

Fig. 3. (A, B) Correlations between the $T 2$ relaxation times and clinical symptoms. The Visual Analog Scale (VAS) score for upper arm pain was not correlated significantly with $\mathrm{T} 2$ values $(r=0.26, p=0.375)$, but was positively correlated with the $\mathrm{T} 2$ ratio $(r=0.54, p=0.047)$.

related with T2 relaxation times $(r=-0.08, p=0.780)$ or with the T2 ratio $(r=-0.28, p=0.335)$. The VAS score for upper arm pain was not significantly correlated with the $\mathrm{T} 2$ relaxation times $(r=0.26, p=0.375)$, but was positively correlated with the T2 ratio $(r=0.54, p=0.047)$ (Fig. 3). 

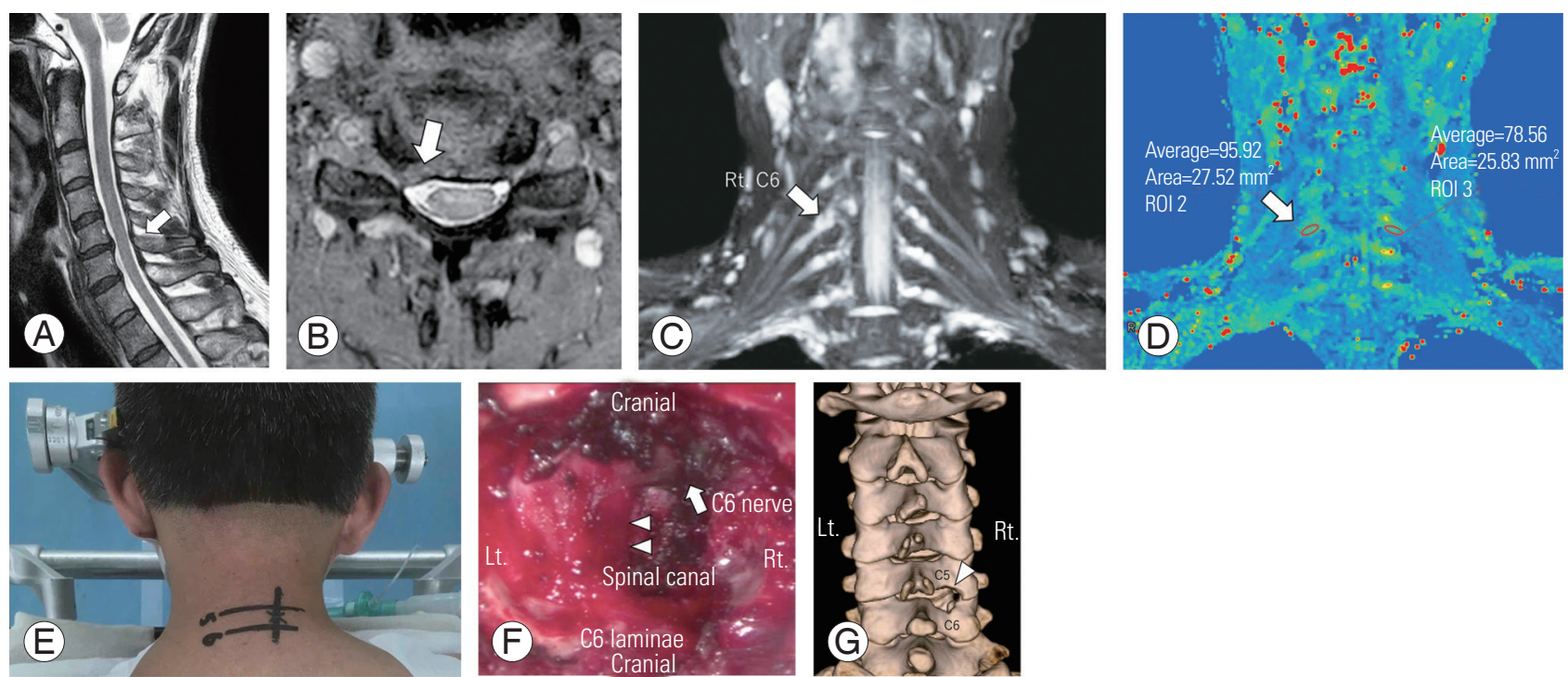

Fig. 4. Images and surgical findings. (A) Herniation of the C5-6 intervertebral disc can be observed from a sagittal plane preoperative magnetic resonance imaging (MRI) (T2-weighted image) of the cervical spine (arrow). (B) C5-6 intervertebral disc herniation on the right side can be observed from a preoperative axial MRI image (arrow) (C) A coronal cervical neurography image shows swelling of the right C6 nerve (arrow). (D) Using cervical T2 mapping in the coronal plane, the T2 relaxation time was found to be significantly prolonged in the right C6 nerve root (arrow). (E) For surgery, the patient was placed in a prone position, with his head secured using threepoint pin fixation devices. The C5-6 intervertebral disc was confirmed using a fluoroscopic C-arm. (F) The inferior margin of the C5 lamina and the superior margin of the C6 lamina were resected, and the medial C5-6 facet joint was removed, exposing the area from the lateral margin of the dura mater (arrowhead) to the C6 nerve bifurcation (arrow). (G) A postoperative three-dimensional computed tomography image of the cervical spine confirmed the fenestration of the inferior margin of the C5 lamina, the superior margin of the C6 lamina, and the medial third of the C5-6 facet joint (arrowhead). Rt, right side; Lt, left side.

\section{Case presentation}

1) Case 1

A 50-year-old man developed pain from the region near his right scapula radiating down the right forearm and causing numbness of his right-hand fingers 2 months before consultation. The pain was resistant to oral medications (tramadol hydrochloride, pregabalin). There was no reduction in muscle strength or sensation. The Japanese Orthopaedic Association (JOA) score was 15.5/17, the VAS score for neck pain was 30, and the VAS scores for upper arm pain and for numbness on the right side were both 100. T2-weighted MRI revealed herniation of the C5-6 intervertebral disk on the right side (Fig. 4A, B).

Neurography revealed swelling of the right C6 nerve (Fig. 4C), and T2 relaxation time of the DRG was significantly prolonged (Fig. 4D). Based on these findings, the patient was diagnosed as having $\mathrm{C} 6$ radiculopathy owing to C5-6 intervertebral disk herniation. The results of MR neurography and T2 mapping were used to explain the pathology and surgical decision to the patient.

The patient was placed in a prone position with his head secured using 3-point pin fixation devices (Fig. 4E). A fluoroscopic C-arm was used to confirm the C5-6 interver- tebral disk. An incision approximately $30 \mathrm{~mm}$ to the right of the C5-6 spinous processes was made, and the muscle fascia and paraspinal muscle groups were bisected. A 16 mm tubular retractor (METRx System Medtronic Sofamor Danek, Minneapolis, MN, USA) was inserted and secured before again confirming the C5-6 intervertebral disk using fluoroscopy. The inferior margin of the C5 lamina and the superior margin of the C6 lamina were removed using a high-speed drill, and the surgical field was expanded laterally to the dura mater. The medial C5-6 facet joint was removed to expose the $\mathrm{C} 6$ nerve bifurcation (Fig. 4F). The area beneath the nerve was explored using a ball probe to confirm the intervertebral disk at that level. Additional unroofing of the foramina was performed up to the distal C6 nerve to achieve decompression. The surgery lasted 160 minutes, with blood loss of $85 \mathrm{~mL}$. The fenestration of the inferior margin of the C5 lamina, the superior margin of the C6 lamina, and the medial third of the C5-6 facet joint were confirmed postoperatively using 3D CT (Fig. $4 \mathrm{G})$. The patient resumed work 1 month after surgery, and the VAS scores (for neck pain, right upper arm pain, and numbness of the right extremity) and JOA score indicated dramatic improvement $(0,0,0 / 100$, and 17 , respectively) 6 months after surgery. 

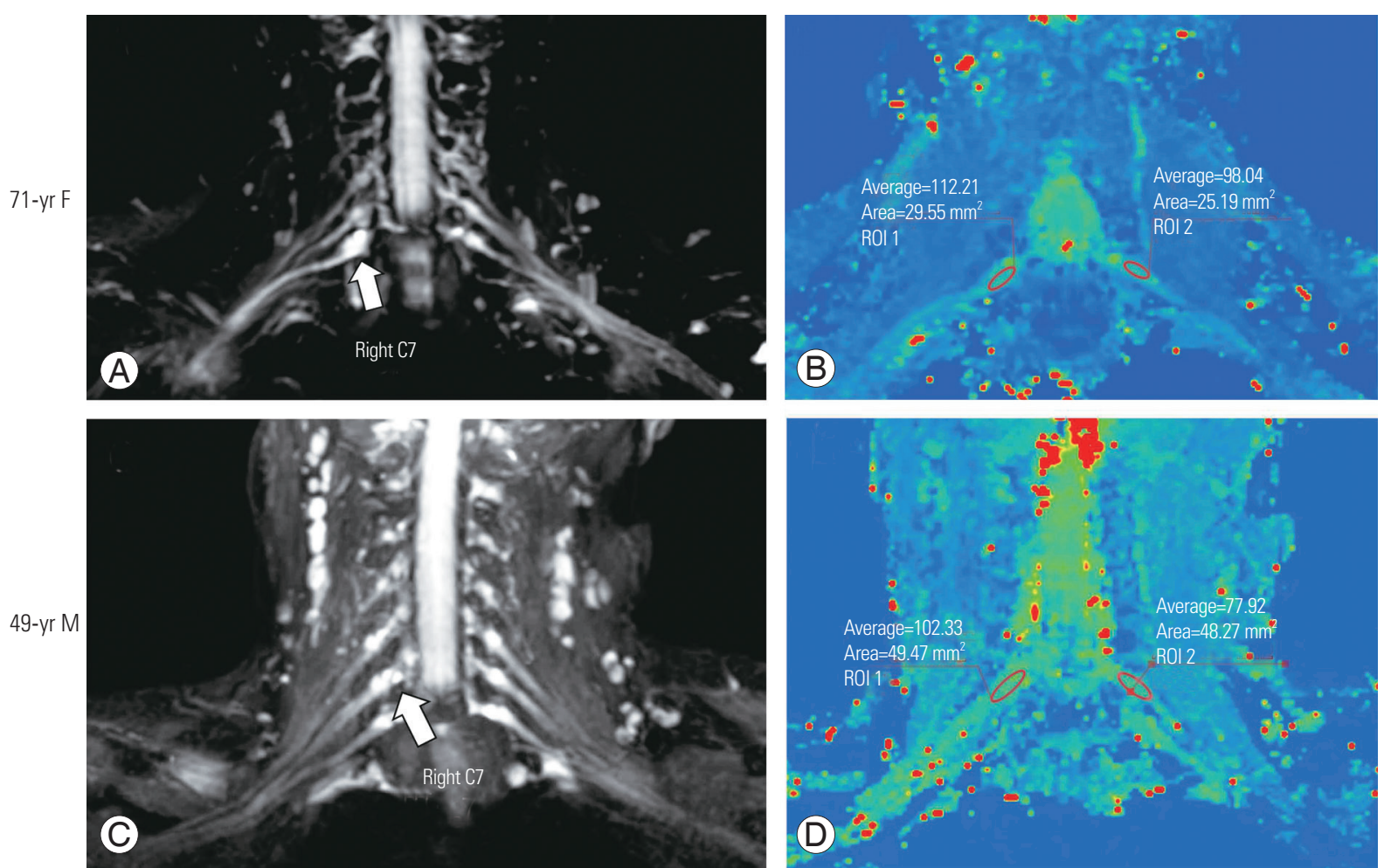

Fig. 5. Neurography and T2 mapping of case 2 (A, B; 71 -year-old woman) and case 3 (C, D; 49-year-old man). (A) Cervical neurography in the coronal plane verifies swelling of the right C7 nerve (arrow). (B) Cervical T2 mapping in the coronal plane indicated a prolonged T2 relaxation time in the right C7 nerve root (right: 112.2, left: 98.0). (C) Cervical neurography in the coronal plane shows swelling of the right C7 nerve (arrow). (D) Cervical T2 mapping in the coronal plane indicated a prolonged T2 relaxation time in the right C7 nerve root (right: 102.3, left: 77.9). ROI, region of interest; $F$, female; $\mathrm{M}$, male.

\section{2) Case 2}

A 71-year-old woman presented with right $\mathrm{C} 7$ radiculopathy owing to C6-7 intervertebral disk herniation. The VAS score for neck pain was 30, and the VAS scores for upper arm pain and for numbness were both 50 on the right side. MR neurography revealed swelling of the right C7 nerve (Fig. 5A). The T2 relaxation time was significantly prolonged in the right C6 DRG (Fig. 5B). Clinical symptoms improved with medication.

\section{3) Case 3}

A 49-year-old man presented with right C7 radiculopathy owing to C6-7 intervertebral disk herniation. The VAS score for neck pain was 0 , and the VAS scores for upper arm pain and for numbness were both 80 on the right side. MR neurography revealed swelling of the right C6 nerve (Fig. 5C), and the DRG T2 relaxation time was significantly prolonged (Fig. 5D). Clinical symptoms improved with microendoscopic surgery.

\section{Discussion}

The cervical nerves of patients with cervical radiculopathy were evaluated quantitatively using SHINKEI-Quant. Neurography revealed cervical nerve swelling, with a longer $\mathrm{T} 2$ relaxation time than the unaffected contralateral nerves. A higher T2 ratio was associated with upper arm pain. Cervical microendoscopic surgery is minimally invasive, making an accurate approach to lesions more difficult $[21,22]$.

The capacity to accurately diagnose cervical disk herniation has markedly improved with the introduction of MRI. 3D MRI and oblique MRI are useful in cases in which definitive diagnoses using clinical symptoms of radiculopathy alone cannot be made [23]. In previous studies, MRI allowed the identification of abnormalities such as herniation of the nucleus pulposus and foramen stenosis in $19 \%$ of asymptomatic subjects [24]. However, MRI can only display morphological abnormalities such as nerve destruction and stenosis but cannot quantitatively assess nerve damage. 
Diffusion tensor imaging (DTI) is a new MRI technique that provides important evidence for changes in tissue microstructure by measuring the anisotropy of water diffusion in vivo [25]. Fractional anisotropy (FA), calculated from DTI, was significantly lower, and apparent diffusion coefficient was significantly higher in entrapped nerve roots than unaffected roots [26]. Previous studies reported that clinical symptoms were highly correlated with FA [26]. Although many studies have reported that DTI is useful in the diagnosis of lumbar lesions [26], DTI of brachial plexus fibers is difficult technically because of small fiber size, respiratory movements, and geometric distortions that can cause various tissue shifts [27].

Although DTI is the only method that can quantitatively evaluate spinal nerves that have branched spinal cords, it is impossible to visualize cervical nerve roots, and currently, no imaging modalities can quantitatively assess the affected cervical nerve roots.

The SHINKEI-Quant technique can provide both MR neurography and T2 mapping simultaneously, which improves the quantitative assessment of neuropathology. Hiwatashi et al. [16,17] reported longer $\mathrm{T} 2$ relaxation times of the roots and ganglia of the brachial plexus and lumbar plexus in patients with CIDP than healthy controls. Moreover, the size of the nerves was larger in patients with CIDP than in healthy subjects. Sato et al. [19] reported that, in patients with lumbar radiculopathy, the $\mathrm{T} 2$ ratio was more diagnostic for radiculopathy than the T2 relaxation time alone. In patients with cervical radiculopathy, cervical neurography exposed enlargement of the affected nerves and prolonged $\mathrm{T} 2$ relaxation times compared with unaffected nerves on the contralateral side. The $\mathrm{T} 2$ ratio correlated highly with upper arm pain and may be a better diagnostic measure than $\mathrm{T} 2$ relaxation time alone to identify those with radiculopathy [18]. T2 relaxation times in affected patients are frequently not reported, and additional cases are needed to verify this observation.

An important study that used SHINEKI-Quant reported that the $\mathrm{T} 2$ relaxation times of the DRG and distal nerves were longer in patients with CIDP (119 ms and $111 \mathrm{~ms}$, respectively) than they were in healthy subjects (101 ms and $85 \mathrm{~ms}$, respectively) [16]. Sato et al. [19] previously reported slightly longer lumbar DRG T2 relaxation times in healthy subjects (mean $=115 \mathrm{~ms}$ ), but in the current study, the T2 relaxation time of the cervical DRG in healthy subjects was consistent with those previous studies (mean=98.5 ms). Karampinos et al. [28] reported that the T2 relaxation times were significantly longer in the DRG of L4 $(78.0 \mathrm{~ms})$ than in more distal parts of the nerve $(59.5 \mathrm{~ms})$. The results reported in this study suggest that the relaxation times might be longer than this.

T2 relaxation times were calculated using only 2 preptimes, which can lead to an incorrect evaluation. T2 relaxation time is usually measured from multislice multiecho spin echo sequences, which might be a shortcoming of MR neurography. We compared T2 relaxation times at 8 time points (TE: 24, 48, 72, 96, 120, 144, 168, and $192 \mathrm{~ms}$ ) and those at 2 time points (SHINKEI-Quant; TE: 36 and $72 \mathrm{~ms}$ ) in the rabbit spinal cord [19]. Using only 2 time points, SHINKEI-Quant calculated that relaxation times were 10-20 ms longer than when 8 time points were used [19]. Using more time points produces a more accurate and consistent assessment.

Because of its multilevel degenerative cervical spine, selective diagnostic radicular block may be a useful differential diagnostic tool for patients with multilevel radicular degeneration of the cervical spine. However, the risks of nerve root damage and vertebral artery perforation from an intradural injection should be considered [29].

There are several limitations in this study. First, the sample size was small. In this study, power analysis confirmed that the T2 relaxation times item was sufficiently high with an actual force of 0.88 in a total of 10 cases as an evidence of $\mathrm{T} 2$ relaxation times for the diagnosis of cervical radiculopathy. The addition of more cases may help to verify the findings presented here. Second, the SHINKEI-Quant was not repeated postoperatively. Third, only $\mathrm{T} 2$ relaxation times of the DRG in the cervical nerves were measured. Fourth, Alrawi et al. [30] reported that patients undergoing surgery for cervical radiculopathy who had an abnormal needle electromyography (EMG) examination preoperatively had better surgical outcomes than those with a normal preoperative EMG, indicating that EMG may discriminate those most likely to benefit from intervention. In this study, EMG examinations were not administered. Fifth, only one clinical outcome such as VAS score was examined.

\section{Conclusions}

The SHINKEI-Quant technique can be used effectively to quantitatively evaluate the compressed nerve roots in patients with cervical radiculopathy. T2 relaxation times of the cervical nerves were significantly prolonged on the 
involved side compared with those of the unaffected side in patients with unilateral radicular symptoms. The VAS score for upper arm pain was positively correlated with the T2 ratio. These results suggest that SHINKEI-Quant is a potential diagnostic tool for cervical nerve entrapment.

\section{Conflict of Interest}

No potential conflict of interest relevant to this article was reported.

\section{References}

1. Radhakrishnan K, Litchy WJ, O'Fallon WM, Kurland LT. Epidemiology of cervical radiculopathy: a population-based study from Rochester, Minnesota, 1976 through 1990. Brain 1994;117(Pt 2):325-35.

2. Carette S, Fehlings MG. Clinical practice: cervical radiculopathy. N Engl J Med 2005;353:392-9.

3. Thoomes EJ, Scholten-Peeters GG, de Boer AJ, et al. Lack of uniform diagnostic criteria for cervical radiculopathy in conservative intervention studies: a systematic review. Eur Spine J 2012;21:1459-70.

4. Friedenberg ZB, Miller WT. Degenerative disc disease of the cervical spine. J Bone Joint Surg Am 1963;45:1171-8.

5. Pech P, Daniels DL, Williams AL, Haughton VM. The cervical neural foramina: correlation of microtomy and CT anatomy. Radiology 1985;155:143-6.

6. Bell GR, Ross JS. Diagnosis of nerve root compression: myelography, computed tomography, and MRI. Orthop Clin North Am 1992;23:405-19.

7. Brown BM, Schwartz RH, Frank E, Blank NK. Preoperative evaluation of cervical radiculopathy and myelopathy by surface-coil MR imaging. AJR Am J Roentgenol 1988;151:1205-12.

8. Takahara T, Hendrikse J, Yamashita T, et al. Diffusion-weighted MR neurography of the brachial plexus: feasibility study. Radiology 2008;249:653-60.

9. Yamashita T, Kwee TC, Takahara T. Whole-body magnetic resonance neurography. N Engl J Med 2009;361:538-9.

10. Yoneyama M, Takahara T, Kwee TC, Nakamura M, Tabuchi T. Rapid high resolution MR neurography with a diffusion-weighted pre-pulse. Magn Reson Med Sci 2013;12:111-9.

11. Ogata H, Yamasaki R, Hiwatashi A, et al. Charac- terization of IgG4 anti-neurofascin 155 antibodypositive polyneuropathy. Ann Clin Transl Neurol 2015;2:960-71.

12. Hiwatashi A, Togao O, Yamashita K, et al. Evaluation of chronic inflammatory demyelinating polyneuropathy: 3D nerve-sheath signal increased with inked rest-tissue rapid acquisition of relaxation enhancement imaging (3D SHINKEI). Eur Radiol 2017;27:447-53.

13. Hiwatashi A, Togao O, Yamashita K, et al. Lumbar plexus in patients with chronic inflammatory demyelinating polyneuropathy: evaluation with $3 \mathrm{D}$ nervesheath signal increased with inked rest-tissue rapid acquisition of relaxation enhancement imaging (3D SHINKEI). Eur J Radiol 2017;93:95-9.

14. Kasper JM, Wadhwa V, Scott KM, Rozen S, Xi Y, Chhabra A. SHINKEI: a novel 3D isotropic MR neurography technique: technical advantages over 3DIRTSE-based imaging. Eur Radiol 2015;25:1672-7.

15. Yoneyama M, Togao O, Hiwatashi A, et al. SHINKEI Quant: simultaneous acquisition of MR neurography and T2 mapping for quantitative evaluation of chronic inflammatory demyelinating polyneuropathy. Proceedings of the ISMRM \& SMRT Annual Meeting and Exhibition; 2016 May 7-13; Singapore. Concord (CA): International Society for Magnetic Resonance in Medicine and the Society for MR Radiographers \& Technologists; 2016.

16. Hiwatashi A, Togao O, Yamashita K, et al. Simultaneous MR neurography and apparent T2 mapping in brachial plexus: evaluation of patients with chronic inflammatory demyelinating polyradiculoneuropathy. Magn Reson Imaging 2019;55:112-7.

17. Hiwatashi A, Togao O, Yamashita K, et al. Lumbar plexus in patients with chronic inflammatory demyelinating polyradiculoneuropathy: evaluation with simultaneous T2 mapping and neurography method with SHINKEI. Br J Radiol 2018;91:20180501.

18. Eguchi Y, Enomoto K, Sato T, et al. Simultaneous MR neurography and apparent T2 mapping of cervical nerve roots before microendoscopic surgery to treat patient with radiculopathy due to cervical disc herniation: preliminary results. J Clin Neurosci 2020;74:213-9.

19. Sato T, Eguchi Y, Norimoto M, et al. Diagnosis of lumbar radiculopathy using simultaneous MR neurography and apparent T2 mapping. J Clin Neurosci 
2020;78:339-46.

20. Brown RW, Cheng YC, Haacke EM, Thompson MR, Venkatesan R. Spin density, T1, and T2 quantification methods in MR imaging. In: Brown RW, Cheng YC, Haacke EM, Thompson MR, Venkatesan R, editors. Magnetic resonance imaging: physical principles and sequence design. 2nd ed. Hoboken (NJ): John Wiley \& Sons Inc.; 2014. p. 637-68.

21. Foley K, Smith M. Microendoscopic discectomy. Tech Neurosurg 1997;3:301-7.

22. Adamson TE. Microendoscopic posterior cervical laminoforaminotomy for unilateral radiculopathy: results of a new technique in 100 cases. J Neurosurg 2001;95(1 Suppl):51-7.

23. Humphreys SC, An HS, Eck JC, Coppes M, Lim TH, Estkowski L. Oblique MRI as a useful adjunct in evaluation of cervical foraminal impingement. J Spinal Disord 1998;11:295-9.

24. Boden SD, McCowin PR, Davis DO, Dina TS, Mark AS, Wiesel S. Abnormal magnetic-resonance scans of the cervical spine in asymptomatic subjects: a prospective investigation. J Bone Joint Surg Am 1990;72:1178-84.
25. Basser PJ, Jones DK. Diffusion-tensor MRI: theory, experimental design and data analysis: a technical review. NMR Biomed 2002;15:456-67.

26. Eguchi Y, Oikawa Y, Suzuki M, et al. Diffusion tensor imaging of radiculopathy in patients with lumbar disc herniation: preliminary results. Bone Joint J 2016;98-B:387-94.

27. Chen YY, Lin XF, Zhang F, et al. Diffusion tensor imaging of symptomatic nerve roots in patients with cervical disc herniation. Acad Radiol 2014;21:338-44.

28. Karampinos DC, Melkus G, Shepherd TM, et al. Diffusion tensor imaging and $\mathrm{T} 2$ relaxometry of bilateral lumbar nerve roots: feasibility of in-plane imaging. NMR Biomed 2013;26:630-7.

29. Anderberg L, Annertz M, Rydholm U, Brandt L, Saveland H. Selective diagnostic nerve root block for the evaluation of radicular pain in the multilevel degenerated cervical spine. Eur Spine J 2006;15:794801.

30. Alrawi MF, Khalil NM, Mitchell P, Hughes SP. The value of neurophysiological and imaging studies in predicting outcome in the surgical treatment of cervical radiculopathy. Eur Spine J 2007;16:495-500. 